The impact of morphology on light transport in cancellous bone

This article has been downloaded from IOPscience. Please scroll down to see the full text article.

2010 Phys. Med. Biol. 554917

(http://iopscience.iop.org/0031-9155/55/17/003)

View the table of contents for this issue, or go to the journal homepage for more

Download details:

IP Address: 131.180.130.114

The article was downloaded on 28/12/2010 at 13:58

Please note that terms and conditions apply. 


\title{
The impact of morphology on light transport in cancellous bone
}

\author{
E Margallo-Balbás ${ }^{1}$, P Taroni $^{2}$, A Pifferi ${ }^{2}$, J H Koolstra $^{3}$, L J v Ruijven ${ }^{3}$ \\ and $\mathbf{P} \mathbf{J}$ French $^{1}$ \\ ${ }^{1}$ Electronic Instrumentation Laboratory, Delft University of Technology, 2628CD Delft, The \\ Netherlands \\ 2 Department of Physics, Politecnico di Milano, 20133 Milan, Italy \\ ${ }^{3}$ Department of Functional Anatomy, Academic Centre for Dentistry Amsterdam (ACTA), \\ University of Amsterdam and VU University, The Netherlands \\ E-mail: e.margallo@tudelft.nl
}

Received 25 January 2010, in final form 7 July 2010

Published 3 August 2010

Online at stacks.iop.org/PMB/55/4917

\begin{abstract}
In recent years, optical techniques based on diffusion approximation have demonstrated their ability to gain rich spectral information about bone. However, these methods normally assume homogeneity, while cancellous bone and marrow form a highly heterogeneous two-phase medium. This paper studies the limitations of this assumption, and quantifies the role of microstructure on long-range transport properties. The propagation of light pulses through trabecular bone is calculated by Monte Carlo simulation of the scattering and absorption in reconstructions of bone samples obtained from x-ray micro tomographic scans. The time-resolved responses are then fitted with the analytical response of a homogeneous material to obtain the apparent transport properties. These properties are used to test different homogenization equations that have been postulated in the past for heterogeneous tissues and to check their accuracy. The results show that nonlinearity and crosstalk between absorption and scattering are statistically significant, although their impact is relatively small. More importantly, we found that the weight of the components is not only affected by their volume fractions, but need to be corrected by other morphologic measures like trabecular spacing or connectivity density. These deviations from the homogeneous assumption are stronger for scattering than for absorption. In conclusion, the average optical properties of cancellous bone are strongly determined by its microstructure, meaning that optical techniques are a valid method for tissue evaluation, but careful consideration of structurerelated perturbation sources is required.
\end{abstract}

(Some figures in this article are in colour only in the electronic version) 


\section{Introduction}

The ability to obtain clinically relevant properties of cancellous bone-such as strength, structure or composition-in a non-ionizing way is important for a wealth of applications. Possibly the most important one is the diagnosis of bone diseases, such as osteoporosis (King et al 2005) or osteomalacia, but applications like local evaluation of the bone tissue bed prior to placement of prosthetic implants (Yang et al 2008) or non-destructive monitoring of the calcification of osteoblasts in tissue engineering applications (Tanaka et al 2008) have also been described in the literature.

Optical methods have been proposed in the past few years to recover information about bone condition both invasively (Ugryumova et al 2004) and non-invasively (Lev et al 2005, Pifferi et al 2004, Takeuchi et al 1997). They are very attractive because of their potential low cost, absence of ionizing effects and their rich informational content, based on the distinct absorption spectra of tissue constituents that make a compositional evaluation in principle possible. A variety of techniques have been developed to extract the scattering and absorption coefficients of tissue from the measurements depending on the specific application and problem geometry. Some of the most sophisticated ones include the different modalities of diffuse optical tomography (DOT) (Hebden and Austin 2007, Zhao et al 2005), but simpler approaches using analytical models have also been successful at determining tissue properties at readily available peripheral locations such as the calcaneus (Pifferi et al 2004).

However accurate these methods can be, the interpretation of their results requires a deep understanding of tissue properties and the mechanisms of light propagation. Cancellous bone, with its volume filling interstitial marrow, forms a highly irregular two-phase medium. The two fractions have very different light transport properties and different refractive indices, but, on the macroscopic scale, i.e. when measuring its average properties, this microstructure is not observable. Homogenization theory has dealt with similar problems in other fields of science and engineering (Pavliotis and Stuart 2008). It typically proceeds by obtaining a field of abstracted average material properties that summarize the underlying microscopic behavior. There are theoretical results that prove the viability of homogenization for different equations, including the diffusion and linear transport equations (Bensoussan et al 1979, Dumas and Golse 2000) that govern tissue optics.

The question of tissue heterogeneity in biomedical optics is not a trivial one. The concern of the biomedical optics community about this issue is endorsed by the many studies devoted to it. In particular, heterogeneity has been studied to determine the consequences of a concentration of absorbers in blood vessels (Liu et al 1995, Firbank et al 1997, van Veen et al 2002, Talsma et al 2001, Hebden et al 2004), for finding different effects, such as crosstalk between absorption and scattering or nonlinearity, and corresponding correction factors. An accurate characterization of these effects is required in order to avoid miscalculations. For the case of trabecular bone, little quantitative work has been done on investigating the basic relations between the optical properties of its two constituents, and the effective coefficients of the resulting two-phase medium. Previous studies investigating cancellous bone have generally assumed it to be a homogeneous medium (Pifferi et al 2004). Other work concentrated on the properties of cortical bone alone (Firbank et al 1993, Ugryumova et al 2004) or lacked abstracted material scattering and absorption properties (Takeuchi et al 1997). This problem is actually shared with other bone tissue probing methods, like quantitative ultrasound, which has suffered from lack of understanding of the physics of wave propagation in the complex trabecular structure (Padilla et al 2006, 2008), which is still a topic of active research.

In this paper, structural properties of trabecular bone determined with micro computed tomography (micro CT) are compared with the optical properties of these specimens computed 
with Monte Carlo software capable of simulating scattering and absorption in specimens with arbitrary geometries consisting of two completely different materials. This effort is applicable to any in vivo diagnostic tool making use of the diffusion approximation to the radiative transfer equation, including direct non-invasive transmission or reflection studies of peripheral bone sites (calcaneus, phalanges, wrist), perioperative measurements during the placement of prosthetic implants, or thinkable non-invasive measurements of bone quality at central locations, such as the hip or the spine, with support from tomographic reconstruction methods. The ability to alter the simulation parameters with complete freedom provides us with powerful insights into the mechanisms of light propagation. We will use this tool to question the assumption of a homogeneous-like aggregation of the properties of bone and marrow accepted in previous works and compare it with effects found in similar contexts for other heterogeneous tissues. The availability of morphologic measures will allow us to check for the influence of structure on long-range transport properties.

\section{Materials and methods}

\subsection{Sample collection and model generation}

Samples of mammal trabecular bone $(N=15)$ were cut into cylindrical shape with $10 \mathrm{~mm}$ diameter and approximately $15 \mathrm{~mm}$ height. Tissue was obtained from two different animal models (domestic pigs and Dutch milk goats) at the Vrije Universiteit Medical Centre, Amsterdam. Animals were in a healthy condition prior to be euthanized in the course of several studies. The site of bone extraction was in all cases unaffected by these parallel investigations. Samples were further classified according to the extraction site: femoral head (FH), femoral condyle (FC) and vertebral body (VB).

Micro CT scans were carried out with a $\mu$ CT40 machine (Scanco Medical AG Zürich). Briefly, the samples were placed in a fluid to avoid dehydration and scanned at a resolution of $18 \mu \mathrm{m}$. Disk-shaped volumes of interest (VOI) with $2 \mathrm{~mm}$ height were selected, centered on the line between emission and collection fibers. This region is thought to be both representative of the full volume and the zone with the strongest influence on light propagation. The bone volume was identified using a segmentation algorithm with a visually determined threshold. Standard morphometrical analysis was performed on the data, and for every specimen the bone volume fraction, the trabecular thickness, the trabecular separation and the connectivity density were determined. The first three were computed using the distance transform method (Hildebrand and Rüegsegger 1997). The connectivity density was calculated using the ConnEuler method (Odgaard and Gundersen 1993).

The segmented dataset was fed to a marching cubes algorithm to compute the surface mesh separating bone from bone marrow. The size of the datasets resulting from this step was reduced by a factor of 6.7 with an algorithm based on edge collapsing, minimizing a quadric error metric (Garland and Heckbert 1997). Great care was taken to ensure topological consistency throughout the mesh preparation, introducing global topology tests before each potential mesh modification. Since the marrow did not offer enough contrast in the micro CT images, the interface between marrow and air had to be added artificially. This was achieved by constructing a cylindrical hull around the specimens, whose axis was aligned with the axis of the samples and whose radius was matched with the radius of the specimens. Due to the irregularity of the shapes, some leeway had to be added to prevent intersections between both interfaces. This resulted in a model that had approximately $30 \%$ more volume than the samples. 


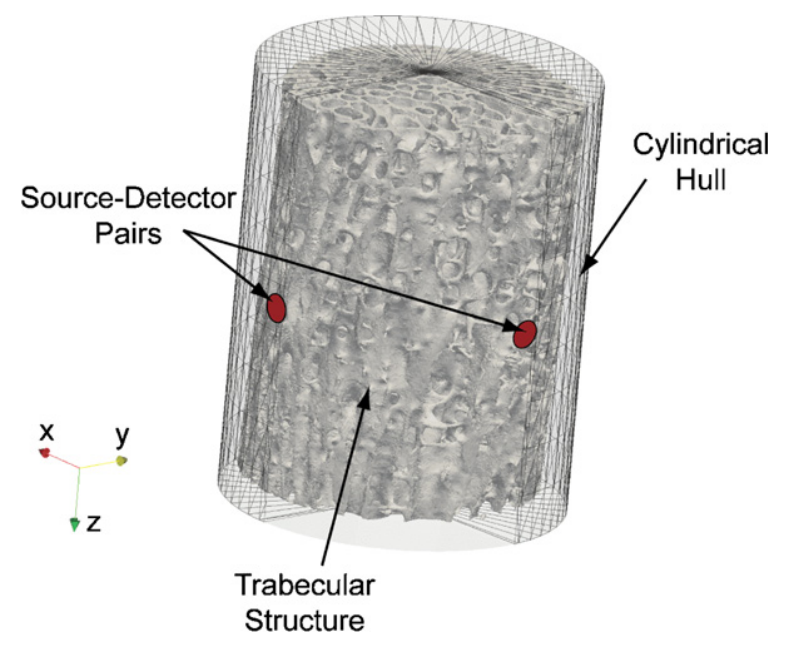

Figure 1. Three-dimensional rendering of the simulation model. Two of the four source-detector pairs that surround the hull defining the external limit of the simulated sample are visible. The detectors are represented as small circles while the sources (hidden) are just under the detectors, pointing toward the cylinder axis. The other two pairs are placed symmetrically relative to the cylinder axis and occluded by the trabecular structure, which is encapsulated within the cylindrical hull.

A ring of four optical source-detector pairs was added to the simulations, placed around the cylinder such that every pair is confronted by another one on the opposite side of the cylinder, i.e. conforming a set of transmission geometries. The sources were modeled as collimated Gaussian profile emitters with a $1 / e$ diameter of $1 \mathrm{~mm}$, while the detectors had circular shape with $1 \mathrm{~mm}$ diameter and unlimited numerical aperture to maximize the number of collected photons. Figure 1 shows a reconstructed model ready for photon tracing by the Monte Carlo core.

\subsection{Monte Carlo simulations and effective coefficients}

The simulations were done using a Monte Carlo code for arbitrarily complex geometries including Russian roulette variance reduction described elsewhere (Margallo-Balbás and French 2007). The code was run at the DAS-3 parallel computing facilities (Bal and Verstoep 2008). For each set of properties, an input file was generated and a large number of photons $\left(N\right.$ ranging from $10^{7}$ to $4 \times 10^{7}$ ) were launched into the geometry, and their paths traced. Spatial distributions of fluence under continuous wave $(\mathrm{CW})$ excitation were registered for the purpose of evaluating the spatial behavior of the field. Photons that hit the detectors were recorded and combined into time-resolved power responses as shown in figure 5. Noise inherent to the stochastic nature of the Monte Carlo method appeared in the curves, but no addition of a dominant Poisson noise contribution was performed. For each set of properties, four simulations corresponding to the four source-detector pairs were produced.

These simulated signals were fitted for each combination of constituent properties using an iterative Levenberg-Marquardt algorithm minimizing the weighted square error between the simulation and the analytical model. The best fit is defined by two average transport coefficients $\mu_{s h}^{\prime}$ and $\mu_{a h}$, which are taken as homogenized parameters. Unless indication to the contrary, the four pairs of homogenized values corresponding to different directions for 
each sample were averaged into a single pair. This is justified by the low directionality found in the results. The analytical model used was the one for the flux across a homogeneous finite cylinder derived under a diffusion approximation to the radiative transport theory with corrections for an unmatched boundary (Arridge et al 1992, Sassaroli et al 1999) expressed as

$\Gamma(t)=\frac{2 \gamma^{2} \mathrm{e}^{-\mu_{a} c t}}{\pi a^{\prime 2} l} \sum_{m=1, \text { odd }}^{\infty} \mathrm{e}^{-\gamma^{2} m^{2} \pi^{2} t / l^{2}} \sum_{n=-\infty}^{+\infty}(-1)^{n} \sum_{\beta_{n}} \beta_{n} \mathrm{e}^{-\gamma^{2} \beta_{n}^{2} t} \frac{J_{n}^{\prime}\left(\beta_{n} a\right) J_{n}\left(\beta_{n} r_{0}\right)}{\left[J_{n}^{\prime}\left(\beta_{n} a^{\prime}\right)\right]^{2}}$.

In this equation, $a^{\prime}$ is the cylinder radius adjusted for the extended boundary condition, which in turn depends on the refractive index mismatch (Arridge et al 1992, Sassaroli et al 1999), a is the physical cylinder radius, $l$ is the cylinder height, $r_{0}$ represents the radial position of the source, $J_{n}$ is the Bessel function of the first kind, $J_{n}^{\prime}$ is the first derivative of $J_{n}, \beta_{n}$ are the roots of $J_{n}\left(\beta_{n} a\right)=0$ and $\gamma$ is given in terms of the absorption and scattering coefficient by (2). This relation assumes that the source and detector are aligned across the center of the cylinder:

$$
\gamma=c\left[3\left(\mu_{a}+\mu_{s}^{\prime}\right)\right]^{-1} \text {. }
$$

Due to the Russian roulette technique for variance reduction, the error weights for the fit were based on the number of collected photons and not on the accumulated energy. The MC signals had an acceptable signal to noise ratio $($ SNR $>10 \mathrm{~dB})$ over more than two decades, depending on the simulated tissue properties. The temporal window of the signals taken into account for the fit was adaptively computed from each curve. The start of the window was taken at the point where the signal was $3 \mathrm{~dB}$ lower than the peak of the curve, with a minimum set at three times the ballistic time of flight. This bound was added to exclude photons that do not conform to the diffusion approximation. The upper temporal limit of the signal window was selected at the point where the signal was $20 \mathrm{~dB}$ lower than the peak value.

For the simulations, we have assumed $n=1.4$ for the marrow and $n=1.6$ for the calcified matrix. These values are broadly consistent with published results (Ascenzi and Fabry 1959, Vo-Dinh 2003) for similar tissues. A single average refractive index is used for the extrapolated boundary. The effect of the extrapolated boundary on the fitted light transport parameters was investigated separately. For this investigation, the transport parameters $\mu_{a}$ and $\mu_{s}$ were set equal for both phases and the refractive indices $\left(n_{m}, n_{b}\right)$ varied over the values $(1.45,1.45)$, $(1.42,1.50)$ and $(1.4,1.6)$, respectively. Generally in this text, the subindices $m$ and $b$ relate to the marrow and bone phases, respectively.

The effect on the fitted parameters was evaluated by plotting them against the set values and then adjusting a second-stage linear model. This model included the option of absorptionscattering crosstalk and the possibility of an intercept. The fit parameters were calculated for each set of refractive indices. The transport coefficients chosen for the calcified matrix are consistent with available experimental results on cortical bone (Firbank et al 1993, Ugryumova et al 2004). The properties of bone marrow are not available in the literature and values consistent with other fat-containing tissues have been adopted, accommodating the range of simulated properties to account for the large expected variability of this parameter.

\subsection{Mix models}

Because of the stochastic nature of the Monte Carlo simulation, statistical inference is applied to the coefficients resulting from the first fitting step described above for the model in equation (1). This means that second-stage linear models are fitted to the values for $\mu_{s h}^{\prime}$, $\mu_{a h}$ resulting from the first fitting step. The role of heterogeneity is evaluated by sequentially constructing second-stage linear models of growing complexity and statistically testing them 
by means of the analysis of their variance. This will be done first on the basis of a single specimen, ignoring the role of geometry, with the goal of understanding the mix mechanisms.

The first mix model is one where the effective properties of the medium are a linear combination of the ones of the constituent phases. Equations (3) and (4) contain a general linear model without crosstalk between scattering and absorption. In the equations, the arbitrary fit coefficients are denoted as $\beta_{a i}$ and $\beta_{s i}$ for absorption and scattering. The model includes a possible non-zero intercept:

$$
\begin{aligned}
& \mu_{a h}=\beta_{a 0}+\beta_{a 1} \mu_{a b}+\beta_{a 2} \mu_{a m} \\
& \mu_{s h}^{\prime}=\beta_{s 0}+\beta_{s 1} \mu_{s b}^{\prime}+\beta_{s 2} \mu_{s m}^{\prime} .
\end{aligned}
$$

The second mix model includes scattering-absorption crosstalk and saturation effects that translate into nonlinear terms and it is contained in equations (5) and (6). Because it is unknown whether these new terms are significant predictors of the homogenized parameters, Akaike's information criterion (AIC) (Akaike 1974) will be used to filter terms with a valuable contribution to the predictive value of the fits:

$$
\begin{aligned}
& \mu_{a h}=\beta_{a 0}+\beta_{a 1} \mu_{s b}^{\prime}+\beta_{a 2} \mu_{s m}^{\prime}+\beta_{a 3} \mu_{a b}+\beta_{a 4} \mu_{a m}+\beta_{a 5} \mu_{a b}^{2}+\beta_{a 6} \mu_{a m}^{2} \\
& \mu_{s h}^{\prime}=\beta_{s 0}+\beta_{s 1} \mu_{s b}^{\prime}+\beta_{s 2} \mu_{s m}^{\prime}+\beta_{s 3} \mu_{a b}+\beta_{s 4} \mu_{a m}+\beta_{s 5} \mu_{s b}^{\prime 2}+\beta_{s 6} \mu_{s m}^{\prime 2} .
\end{aligned}
$$

For the case of multiple specimens, it is necessary to introduce model weights that are related to morphologic parameters. Firstly, a linear model considering the role of volume fractions alone as specified in equations (7) and (8) is evaluated. In these equations, $B V F$ represents the volume fraction of the calcified matrix:

$$
\begin{aligned}
& \mu_{a h}=\beta_{a 0}+\beta_{a 1} B V F \mu_{a b}+\beta_{a 2}(1-B V F) \mu_{a m} \\
& \mu_{s h}^{\prime}=\beta_{s 0}+\beta_{s 1} B V F \mu_{s b}^{\prime}+\beta_{s 2}(1-B V F) \mu_{s m}^{\prime} .
\end{aligned}
$$

Finally, a more complex model including other morphologic parameters will be tested. Trabecular connectivity density, spacing and thickness enter as additional moderators of the individual phases that modify the weights given by the volume fractions alone. Equations (9) and (10) contain this speculative model with a variety of additional structural parameters. Tb.Sp. represents the average intertrabecular spacing, Tb.Th. is the average trabecular thickness and conn represents the connectivity density. AIC (Akaike 1974) was also used in this case to remove terms without a significant contribution to the fit:

$$
\begin{gathered}
\mu_{a h}=\beta_{a 0}+\left[\beta_{a 1} B V F+\beta_{a 2} \text { Tb.Sp. }+\beta_{a 3} \text { Tb.Th. }+\beta_{a 4} \text { conn }\right] \mu_{a b} \\
+\left[\beta_{a 5}(1-B V F)+\beta_{a 6} \text { Tb.Sp. }+\beta_{a 7} \text { Tb.Th. }+\beta_{a 8} \text { conn }\right] \mu_{a m} \\
\mu_{s h}^{\prime}=\beta_{s 0}+\left[\beta_{s 1} B V F+\beta_{s 2} \text { Tb.Sp. }+\beta_{s 3} \text { Tb.Th. }+\beta_{s 4} \text { conn }\right] \mu_{s b}^{\prime} \\
+\left[\beta_{s 5}(1-B V F)+\beta_{s 6} \text { Tb.Sp. }+\beta_{s 7} \text { Tb.Th. }+\beta_{s 8} \text { conn }\right] \mu_{s m}^{\prime} .
\end{gathered}
$$

\section{Results}

Figure 2 shows a typical slice of the micro CT. Only the calcified matrix has meaningful $\mathrm{x}$-ray absorption and is visible in the scans. Marrow, being a soft tissue, appears as a lowdensity medium, often indistinguishable from the surrounding fluid. The figure clearly shows 


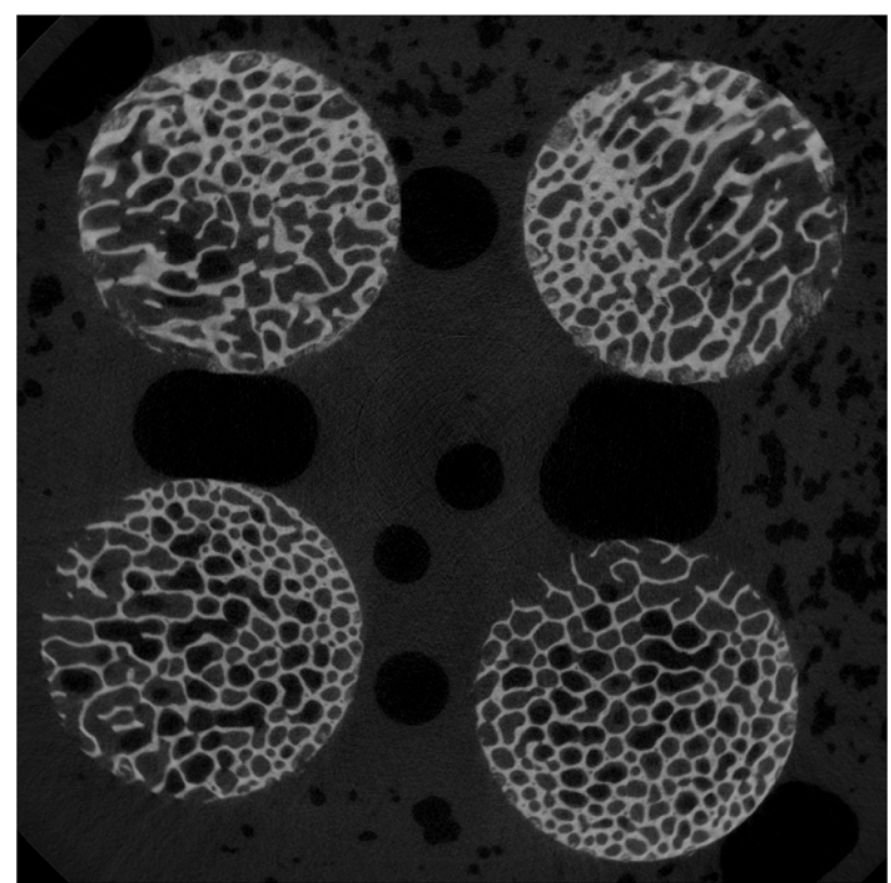

Figure 2. Slice of a micro CT scan of four bone samples showing the complex microstructure of the calcified matrix.
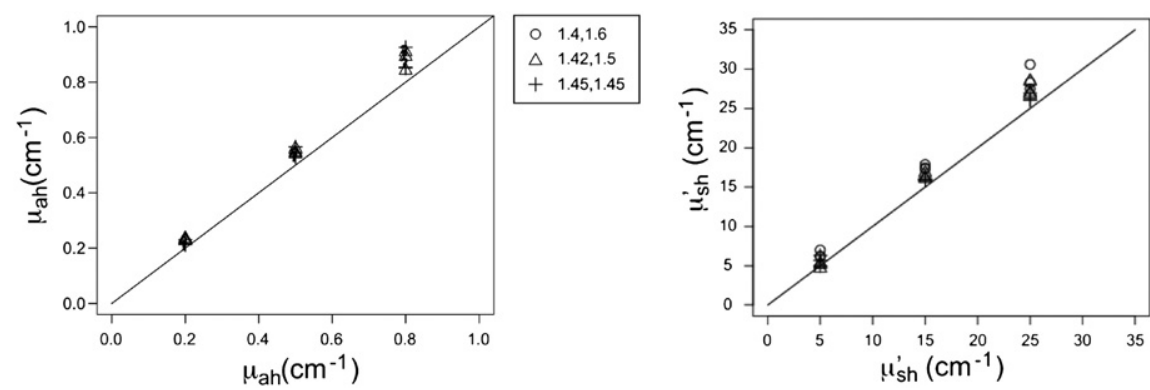

○ 1.4 .1 .6 $\triangle 1.42 .1 .5$ $+1.45 .1 .45$

Figure 3. Actual versus fitted absorption (left) and scattering (right) coefficients for the three simulated values of refractive index mismatch.

the complex and random morphology that characterizes cancellous bone. Table 1 contains the descriptive statistics of the morphologic parameters calculated for all the 15 specimens considered. The statistical parameters of the microstructure-volume fraction, intertrabecular distance, etc - are seen to present a large spread over the samples.

Figure 3 shows the effect of the extrapolated boundary on the fitted values for absorption and scattering. The continuous line represents the identity. A linear regression was performed on the fitted values, whose results are contained in table 2. Firstly, the intercepts are not statistically significant. Secondly, the crosstalk terms also lack statistical significance, with the only exception of the absorption term in the scattering regression for the $(1.4,1.6)$. For 


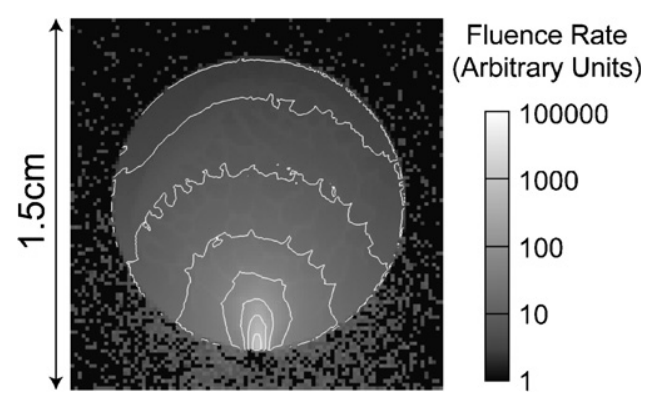

Figure 4. Simulated map of fluence rate distribution within a section of a bone specimen. The transport parameters used were $\mu_{s m}^{\prime}=5.0 \mathrm{~cm}^{-1} \mu_{a m}=0.2 \mathrm{~cm}^{-1}, \mu_{s b}^{\prime}=17.5 \mathrm{~cm}^{-1}, \mu_{a b}=$ $0.2 \mathrm{~cm}^{-1}$. The source can be seen at the bottom. The white lines represent curves of constant fluence rate and are regularly spaced in the logarithmic scale.

Table 1. Descriptive statistics for the morphological parameters computed on the sample collection $(N=15)$ and used for the study.

\begin{tabular}{lcc}
\hline & Mean & Std deviation \\
\hline BV/TV & 0.34 & 0.12 \\
Conn.-Dens. & 33 & 37 \\
Tb.Sp. & $0.52(\mathrm{~mm})$ & $0.23(\mathrm{~mm})$ \\
Tb.Th. & $0.19(\mathrm{~mm})$ & $0.096(\mathrm{~mm})$ \\
\hline
\end{tabular}

the case where both media are matched, there is a slight overestimation of the parameters (4\% for scattering and $6 \%$ for absorption). Refractive index mismatch seems to strengthen this overestimation for scattering, with the systematic error for the pair $(1.4,1.6)$ at $11 \%$.

Figure 4 shows a plot of the simulated fluence rate map in tissue for the plane defined by the array of sources and detectors under $\mathrm{CW}$ excitation. This simulation corresponds to $10^{7}$ photons being launched from the source, which can be seen at the bottom of each graph. The central disk-shaped light distribution is caused by transport in tissue, while photons outside of the cylinder are due to the small, but non-zero interaction coefficient postulated for the surrounding medium. Figure 5 shows two time-resolved power responses. Set 1 had optical properties $\mu_{s m}^{\prime}=10 \mathrm{~cm}^{-1}, \mu_{a m}=0.8 \mathrm{~cm}^{-1}, \mu_{s b}^{\prime}=24.5 \mathrm{~cm}^{-1}, \mu_{s m}^{\prime}=0.2 \mathrm{~cm}^{-1}$ and set 2 had properties $\mu_{s m}^{\prime}=10 \mathrm{~cm}^{-1}, \mu_{a m}=0.4 \mathrm{~cm}^{-1}, \mu_{s b}^{\prime}=14 \mathrm{~cm}^{-1}, \mu_{s m}^{\prime}=0.2 \mathrm{~cm}^{-1}$. Each set consists of four curves corresponding to the four possible source-detector pairs. Since the four curves appear on top of each other, there is no apparent direction-related dependence. This was confirmed after fitting the curves with the model.

The parameters computed by fitting the data for specimen number 1 to the analytical diffusion model for a finite cylinder are shown in figure 6 . The values of the coefficients $\mu_{a m}$, $\mu_{s m}^{\prime}, \mu_{a b}$ and $\mu_{s b}^{\prime}$ were chosen using a mixed experimental design composed of a combination of a full factorial design with Latin hypercube sampling. The full factorial design involved $2^{4}=16$ simulations per model. It is suitable to detect and precisely quantify variable interactions. The Latin hypercube samples represent nine additional points within the variable space. The same optical properties were used for the simulations of all bone samples. For the samples picked in the factorial design, the coefficients $\mu_{a m}, \mu_{s m}^{\prime}, \mu_{a b}$ and $\mu_{s b}^{\prime}$ were allowed to vary among the values $\left(0.02 \mathrm{~mm}^{-1}, 0.08 \mathrm{~mm}^{-1}\right)\left(0.02 \mathrm{~mm}^{-1}, 0.04 \mathrm{~mm}^{-1}\right),\left(5 \mathrm{~mm}^{-1}\right.$, $\left.10 \mathrm{~mm}^{-1}\right)$ and $\left(25 \mathrm{~mm}^{-1}, 30 \mathrm{~mm}^{-1}\right)$, respectively. A wide range of properties is obtained, 


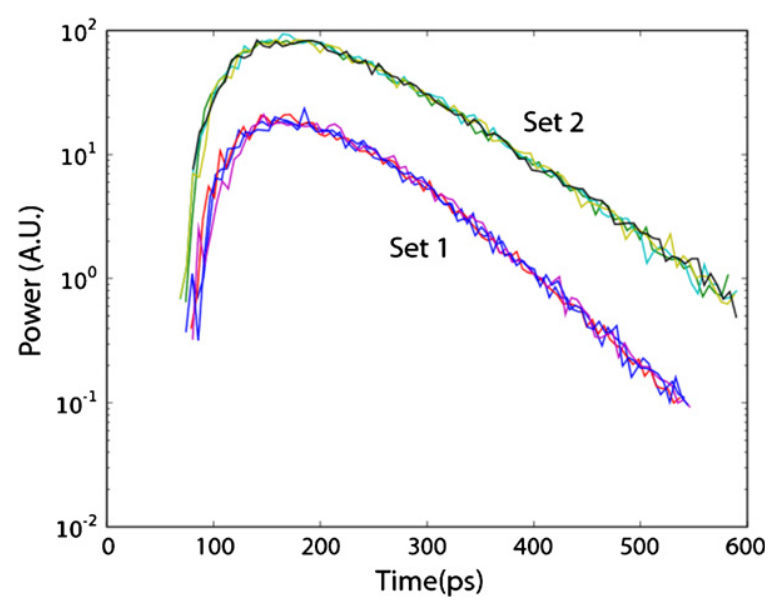

Figure 5. Simulated time-resolved transmission curves across a single specimen for two sets of optical parameters. The four possible source-detector combinations are overlaid in the graph and represented by different line styles. The vertical axis shows the power falling on the detectors in arbitrary units, the horizontal axis holds the time in picoseconds.

Table 2. Linear regression on the fitted transport coefficients $(N=35)$ for a bone model with refractive index mismatches between marrow and bone and otherwise homogeneous transport coefficients. The sets of indices of marrow and bone $\left(n_{m}, n_{b}\right)$ were I $(1.4,1.6)$, II $(1.42,1.5)$ and III $(1.45,1.45)$.

\begin{tabular}{|c|c|c|c|c|}
\hline \multicolumn{5}{|c|}{ Regression for $\mu_{s h}^{\prime}$} \\
\hline & Estimate & Std error & $t$ value & $\operatorname{Pr}(>|t|)$ \\
\hline Intercept & $-0.1\left(\mathrm{~cm}^{-1}\right)$ & $0.3\left(\mathrm{~cm}^{-1}\right)$ & -0.4 & 0.7 \\
\hline$\mu_{\mathrm{s}}^{\prime}(\mathrm{I})$ & 1.11 & 0.02 & 55.6 & $<10^{-4}$ \\
\hline$\mu_{\mathrm{s}}^{\prime}(\mathrm{II})$ & 1.11 & 0.02 & 55.3 & $<10^{-4}$ \\
\hline$\mu_{\mathrm{s}}^{\prime}(\mathrm{III})$ & 1.04 & 0.02 & 52.0 & $<10^{-4}$ \\
\hline$\mu_{\mathrm{a}}(\mathrm{I})$ & 1.9 & 0.6 & 3.0 & $<10^{-2}$ \\
\hline$\mu_{\mathrm{a}}(\mathrm{II})$ & -0.5 & 0.6 & -0.9 & 0.4 \\
\hline$\mu_{\mathrm{a}}(\mathrm{III})$ & 0.4 & 0.6 & 0.7 & 0.5 \\
\hline \multicolumn{5}{|c|}{ Regression for $\mu_{a h}$} \\
\hline Intercept & $0.025\left(\mathrm{~cm}^{-1}\right)$ & $0.014\left(\mathrm{~cm}^{-1}\right)$ & 1.8 & 0.08 \\
\hline$\mu_{\mathrm{a}}(\mathrm{I})$ & 1.08 & 0.03 & 39.0 & $<10^{-4}$ \\
\hline$\mu_{\mathrm{a}}(\mathrm{II})$ & 1.04 & 0.03 & 37.4 & $<10^{-4}$ \\
\hline$\mu_{\mathrm{a}}(\mathrm{III})$ & 1.06 & 0.03 & 38.5 & $<10^{-4}$ \\
\hline$\mu_{\mathrm{s}}^{\prime}(\mathrm{I})$ & -0.001 & 0.001 & -1.1 & 0.26 \\
\hline$\mu^{\prime} \mathrm{s}(\mathrm{II})$ & 0.001 & 0.001 & 0.7 & 0.50 \\
\hline$\mu_{\mathrm{s}}^{\prime}(\mathrm{III})$ & -0.001 & 0.001 & -1.6 & 0.14 \\
\hline
\end{tabular}

that is compatible with expected values for tissue. The results for a linear regression based on the model described in equations (3) and (4) are shown in table 3. These linear models for scattering and absorption capture the two most important relationships between the properties of the homogenized model and its constituent phases. The estimated coefficients for both pairs 


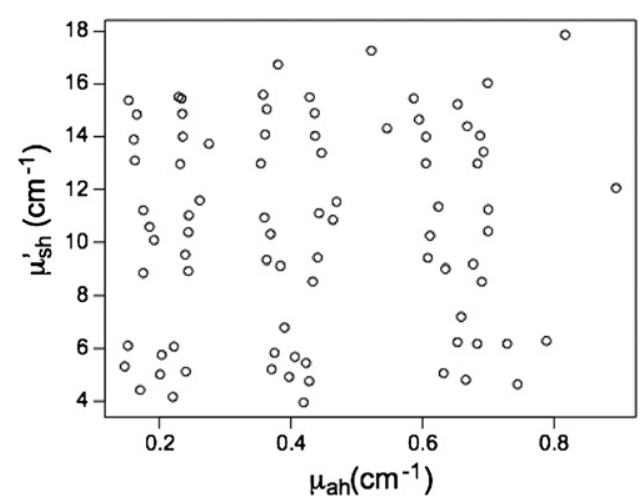

Figure 6. Scatter plot of the coefficients computed by fitting the analytical finite cylinder model to the time-resolved curves generated using $\mathrm{MC}$ for specimen number 1.

Table 3. Linear regression on the homogenized absorption and scattering coefficients $\mu_{s h}^{\prime}$ (top) and $\mu_{a h}$ (bottom) for a simple homogeneous mix equation set.

\begin{tabular}{llccc}
\hline \multicolumn{5}{c}{ Regression for $\mu_{s h}^{\prime}$} \\
\hline & Estimate & Std error & $t$ value & $\operatorname{Pr}(>|t|)$ \\
\hline Intercept & $0.93\left(\mathrm{~cm}^{-1}\right)$ & $0.28\left(\mathrm{~cm}^{-1}\right)$ & 3.2 & $<\mathbf{2 \times \mathbf { 1 0 } ^ { - 3 }}$ \\
\hline$\mu_{s b}^{\prime}$ & 0.20 & 0.01 & 14.4 & $<\mathbf{1 0}^{-4}$ \\
$\mu_{s m}^{\prime}$ & 0.95 & 0.01 & 65.3 & $<\mathbf{1 0}^{-4}$ \\
& \multicolumn{5}{c}{ Regression for $\mu_{a h}$} \\
\hline Intercept & $0.036\left(\mathrm{~cm}^{-1}\right)$ & $0.008\left(\mathrm{~cm}^{-1}\right)$ & 4.4 & $<\mathbf{1 0}^{-4}$ \\
\hline$\mu_{a b}$ & 0.34 & 0.02 & 14.1 & $<\mathbf{1 0}^{-\mathbf{4}}$ \\
$\mu_{a m}$ & 0.656 & 0.008 & 84.5 & $<\mathbf{1 0}^{-4}$ \\
\hline
\end{tabular}

of predictors are extremely significant, as visible in their $t$ - and $p$-values. The models are able to explain $98.1 \%$ of the variance $\left(\operatorname{adj} . R^{2}=0.981\right)$ in the homogenized scattering coefficient and $98.7 \%$ for the case of absorption (adj. $R^{2}=0.987$ ).

Both regressions pass approximately through the origin. The intercept value for the scattering model is not of statistical relevance, while the value for absorption is significant. However, in the latter case, the intercept is less than $10 \%$ of the mean value of the recovered coefficients. The predictive value of these intercept terms with regard to explained variance is limited, with a reduction of residual variance of $5 \%$ for scattering and $10 \%$ for absorption upon introduction.

The fit of the extended model in equations (5) and (6) to the data was significantly better $\left(p<10^{6}\right)$ than the fit for the linear model. Table 4 shows the regression results. The residual variance was reduced by approximately a factor of 3 in scattering and by $32 \%$ in absorption. Again, both linear scattering and absorption terms have extremely significant coefficients for the regression of the respective homogenized parameters, as expected. In the case of absorption, we find that the scattering crosstalk term for the marrow phase $\left(\mu_{s m}^{\prime}\right)$ is clearly significant. Looking at the average scattering coefficient $\mu_{s h}^{\prime}$, we see that the second-order term for bone is highly significant while the one for marrow is not. The crosstalk terms from 
Table 4. Linear regression on the homogenized scattering $\left(\mu_{s h}^{\prime}\right.$, top) and absorption $\left(\mu_{a h}\right.$, bottom) coefficients for a mix equation set including saturation effects and absorption-scattering crosstalk.

\begin{tabular}{lccrc}
\hline \multicolumn{5}{c}{ Regression for $\mu_{s h}^{\prime}$} \\
\hline & Estimate & Std error & $t$ value & $\operatorname{Pr}(>|t|)$ \\
\hline Intercept & $0.34\left(\mathrm{~cm}^{-1}\right)$ & $0.25\left(\mathrm{~cm}^{-1}\right)$ & 1.4 & 0.18 \\
\hline$\mu_{s b}^{\prime}$ & 0.21 & 0.01 & 21.9 & $<\mathbf{1 0}^{-4}$ \\
$\mu_{a b}$ & -0.80 & 0.39 & -2.0 & $<\mathbf{5} \times \mathbf{1 0}^{-\mathbf{2}}$ \\
$\mu_{s m}^{\prime}$ & 1.31 & 0.04 & 34.0 & $<\mathbf{1 0}^{-\mathbf{4}}$ \\
$\mu_{s b}^{\prime 2}$ & -0.030 & 0.003 & -9.6 & $<\mathbf{1 0}^{-\mathbf{4}}$ \\
$\mu_{a m}$ & 0.20 & 0.12 & 1.6 & 0.11 \\
& & Regression for $\mu_{a h}$ & & \\
\hline Intercept & $0.048\left(\mathrm{~cm}^{-1}\right)$ & $0.008\left(\mathrm{~cm}^{-1}\right)$ & 5.9 & $<\mathbf{1 0}^{-\mathbf{4}}$ \\
\hline$\mu_{a b}$ & 0.34 & 0.02 & 15.3 & $<\mathbf{1 0}^{-4}$ \\
$\mu_{a m}$ & 0.66 & 0.01 & 92.1 & $<\mathbf{1 0}^{-4}$ \\
$\mu_{s m}^{\prime}$ & -0.0022 & 0.0006 & -4.0 & $<\mathbf{2} \times \mathbf{1 0}^{-\mathbf{4}}$ \\
\hline
\end{tabular}

absorption to scattering contribute in the sense of the AIC, but their coefficients do not reach the level of statistical significance. The situation with the intercepts is analogous to the one in the previous model.

The linear model contained in equations (7) and (8) was adjusted to the parameters gained from fitting the finite cylinder model to simulated curves for 15 samples using the material optical properties dictated by the experimental design described above. The regression achieved an adjusted determination coefficient of $R^{2}=0.89$ for the scattering component and $R^{2}=0.97$ for absorption. This means that this simple model captures a very important part of the variance in the regressands. Nonetheless, the difference in behavior between both variables is apparent, with absorption conforming better to the homogeneous mix principle. Table 5 contains the results of the regression. The intercepts are in both cases small compared to the values of the fitted parameters and only significantly different from zero in the case of absorption, similarl to results on a single sample. Both phases have a very significant contribution to the corresponding homogenized values. Residual standard errors are abnormally high compared to the ones obtained for a single specimen. A more careful study of these errors was conducted to investigate the sources of misfit. Figure 7 contains a box plot of the residuals classified according to the specimen they correspond to. This plot reveals a strong structure in the residuals related to the geometry, with each individual specimen displaying a similar spread, with a systematic error added on top of it. This deviation is stronger for the case of scattering, while absorption shows a more limited geometry-related organization.

Given the speculative nature of the more complex model contained in equations (9) and (10), AIC is used again to filter only terms that have a meaningful contribution to the model predictive value. This yields the linear regression summarized in table 6 . We observe that several new terms achieve a high level of significance. In the case of scattering, the connectivity density and the trabecular spacing play an important role as moderators to the scattering of both phases. The trabecular thickness was not significant as a moderator. Regarding absorption, the trabecular spacing and connectivity density enter as significant moderators to both phases, 

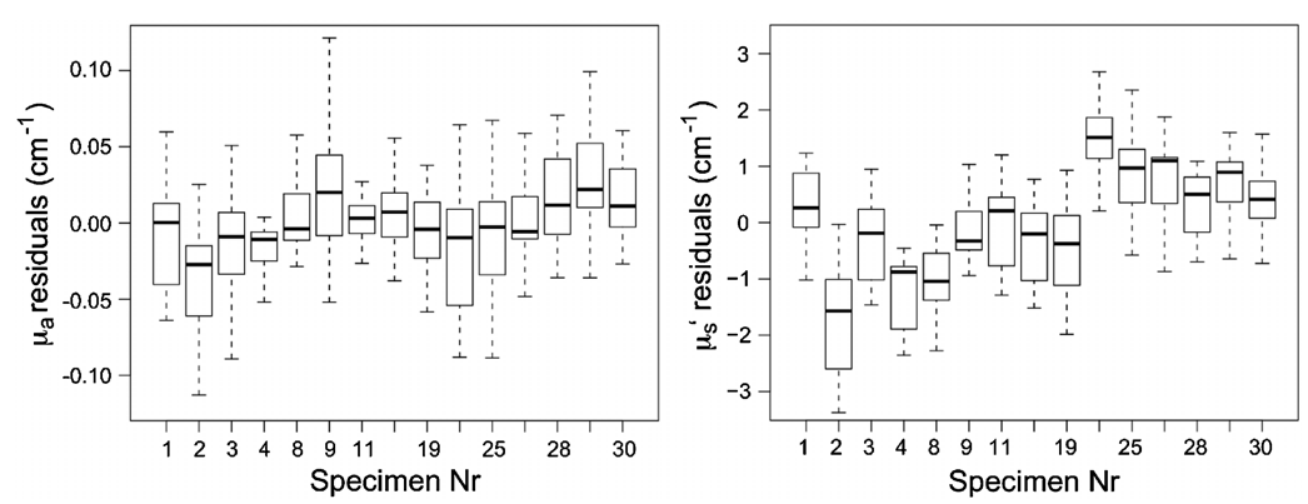

Figure 7. Box plots of the residuals for absorption (left) and scattering (right) against the specimen number for the fit including only the volume fraction.

Table 5. Linear regression on the homogenized scattering ( $\mu_{s h}^{\prime}$, top) and absorption ( $\mu_{a h}$, bottom) coefficients on the full sample set for a model taking into account bone volume fraction alone.

\begin{tabular}{lllrc}
\hline \multicolumn{5}{c}{ Regression for $\mu_{s h}^{\prime}$} \\
\hline Intercept & Estimate & Std error & $t$ value & $\operatorname{Pr}(>|t|)$ \\
\hline$B V F \mu_{s b}^{\prime}$ & $0.38\left(\mathrm{~cm}^{-1}\right)$ & $0.27\left(\mathrm{~cm}^{-1}\right)$ & 1.4 & 0.17 \\
$(1-B V F) \mu_{s m}^{\prime}$ & 1.19 & 0.02 & 51.4 & $<\mathbf{1 0}^{-4}$ \\
& \multicolumn{5}{c}{ Regression for $\mu_{a h}$} \\
\hline Intercept & $0.070\left(\mathrm{~cm}^{-1}\right)$ & $0.006\left(\mathrm{~cm}^{-1}\right)$ & 11.0 & $<\mathbf{1 0}^{-4}$ \\
\hline$B V F \mu_{a b}$ & 0.71 & 0.045 & 15.8 & $<\mathbf{1 0}^{-4}$ \\
$(1-B V F) \mu_{a m}$ & 0.98 & 0.01 & 109.7 & $<\mathbf{1 0}^{-4}$ \\
\hline
\end{tabular}

while trabecular thickness is only significant as a moderator to the properties of the marrow phase.

The addition of the new elements lead to the goodness of fit significantly improving for both sub models, with adjusted determination coefficients $R^{2}=0.962$ for scattering and $R^{2}=$ 0.988 for absorption. This improvement is consonant with a reduction in the non-explained variance by $64 \%$ for scattering and $56 \%$ for absorption. The level of the residuals, albeit smaller, is still higher than the one for a single sample, but no dependence with the specimen number is observable. This can be observed in the box plot in figure 8 .

\section{Discussion}

In this paper we have investigated the relation between the properties of the two phases that fill the trabecular space of bones and their homogenized parameters by means of Monte Carlo simulations on reality-based models. The approach, novel to the best of our knowledge, allowed isolation of the individual contributions of geometry and constituent properties. The results of this study show that the expected refractive index mismatch between the calcified 

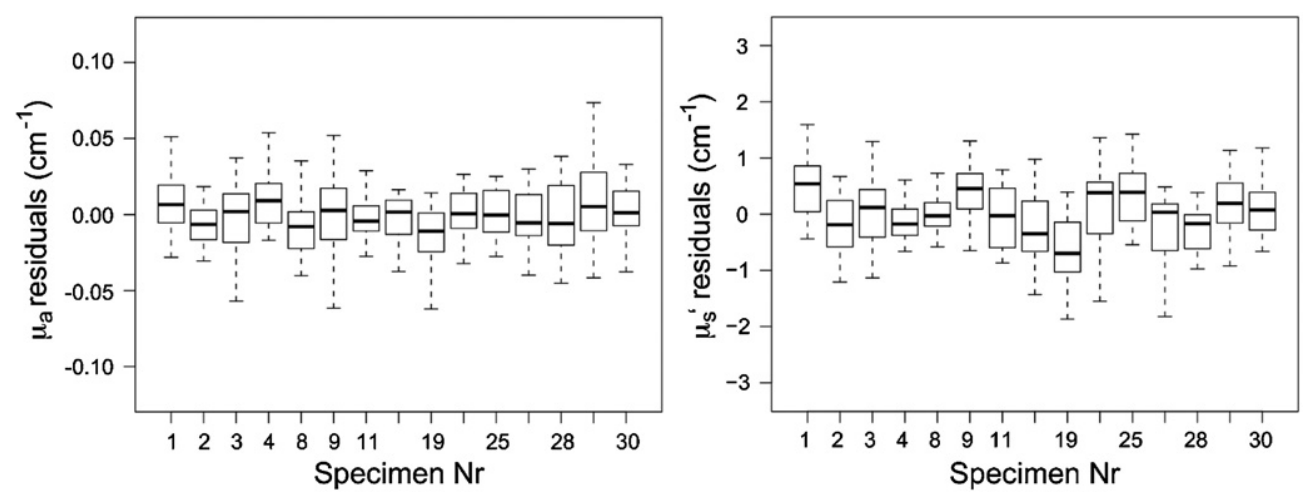

Figure 8. Box plots of the residuals for absorption (left) and scattering (right) against the specimen number for the fits including morphologic parameters other than the volume fractions.

Table 6. Results for a linear regression of the homogenized scattering ( $\mu_{s h}^{\prime}$, top) and absorption ( $\mu_{a h}$, bottom) coefficients on the set of different specimens for a model taking into account other morphologic parameters on top of the volume fractions.

\begin{tabular}{lclrl}
\hline \multicolumn{5}{c}{ Regression for $\mu_{s h}^{\prime}$} \\
\hline Estimate & Std error & $t$ value & $\operatorname{Pr}(>|t|)$ \\
\hline Intercept & $-1.99\left(\mathrm{~cm}^{-1}\right)$ & $0.21\left(\mathrm{~cm}^{-1}\right)$ & -9.6 & $<\mathbf{1 0}^{-4}$ \\
\hline BVF $\mu_{s b}^{\prime}$ & 1.18 & 0.02 & 70.9 & $<\mathbf{1 0}^{-4}$ \\
$(1-B V F) \mu_{s m}^{\prime}$ & 0.95 & 0.03 & 32.8 & $<\mathbf{1 0}^{-4}$ \\
Tb.Sp. $\mu_{s b}^{\prime}$ & -0.19 & 0.01 & -14.2 & $<\mathbf{1 0}^{-4}$ \\
Tb.Sp. $\mu_{s m}^{\prime}$ & 0.19 & 0.03 & 5.5 & $<\mathbf{1 0}^{-4}$ \\
conn $\mu_{s m}^{\prime}$ & $8.410^{-4}$ & $1.510^{-4}$ & 5.7 & $<\mathbf{1 0}^{-4}$ \\
& & Regression for $\mu_{a h}$ & & \\
\hline Intercept & $0.031\left(\mathrm{~cm}^{-1}\right)$ & $0.005\left(\mathrm{~cm}^{-1}\right)$ & 6.4 & $<\mathbf{1 0}^{-4}$ \\
\hline BVF $\mu_{a b}$ & 0.79 & 0.04 & 19.1 & $<\mathbf{1 0}^{-4}$ \\
$(1-B V F) \mu_{a m}$ & 1.15 & 0.01 & 93.4 & $<\mathbf{1 0}^{-4}$ \\
conn $\mu_{a b}$ & $1.510^{-3}$ & $210^{-4}$ & 7.2 & $<\mathbf{1 0}^{-4}$ \\
conn $\mu_{a m}$ & $-10^{-3}$ & $10^{-4}$ & -9.7 & $<\mathbf{1 0}^{-4}$ \\
Tb.Sp. $\mu_{a b}$ & 0.17 & 0.03 & 6.6 & $<\mathbf{1 0}^{-4}$ \\
Tb.Sp. $\mu_{a m}$ & -0.12 & 0.02 & -5.2 & $<\mathbf{1 0}^{-4}$ \\
Tb.Th. $\mu_{a m}$ & -0.18 & 0.04 & -4.1 & $<\mathbf{1 0}^{-4}$ \\
\hline
\end{tabular}

matrix of cancellous bone and the marrow filling the void space in the microstructure can have an impact on the average properties computed using diffuse models. This mismatch could translate into a potential overestimation of the coefficients, which is stronger in the case of scattering, but the effect seems minor for the structures and properties investigated. We have also found that a strong refractive index contrast between phases can induce crosstalk from absorption into scattering. This crosstalk had, in any case, a very small contribution to variance explanation. 
The spatial distribution of the fluence rate has been shown to vary slowly at scales much larger than the microstructure under $\mathrm{CW}$ excitation, while the difference in the fluence rate between marrow and calcified matrix adds a high frequency field on the average result. This result is typical for fields in homogenization studies and supportive of the methodology. The introduction of heterogeneity in terms of scattering and absorption between both media has repercussions for the homogenized parameters. The results for a model based on a linear combination of the properties of the individual tissue constituents delivered large determination coefficients for both absorption and scattering. The value of intercepts in all models was minor, as would be expected based on physical arguments. Indeed, for a clear medium in both bone and marrow, the resulting average should also be clear, although the refractive index mismatch disturbs this reasoning slightly.

The fit results imply that the simple linear mix equations (3) and (4) are appropriate, but the results could be due to the fact that the postulated properties for both absorption and scattering fluctuate over a large range of values that might not be representative of clinically relevant situations. For a different set of inputs-e.g. uniform values for the scattering propertiessecond-order effects such as the ones described in the literature (Liu et al 1995, Firbank et al 1997, van Veen et al 2002, Talsma et al 2001, Hebden et al 2004) could play a role. Indeed, a more complex model including saturation effects and crosstalk proved to be statistically better at predicting our simulation results. Even accepting a simple linear mix without crosstalk between scattering and absorption, we found that the weights are not purely determined by the volume fractions of the corresponding phases as would be expected if the scatterers and absorbers were to act as homogeneously distributed. They are actually also influenced by morphologic parameters such as intertrabecular distance or connectivity density.

In conclusion, this study shows that the average optical parameters of trabecular bone are determined for the most part by the properties of its two constituent phases and the fraction of the total volume occupied by the calcified matrix. It also quantifies the relative contributions of different structural perturbation sources to the mix equations, from which correction factors can be derived. This work proves that light propagation in the trabecular structure of bone is well understood and that it can be analyzed under the diffusion approximation. These results represent an improvement of our understanding of the role of heterogeneity for this specific tissue type and they sustain the reconstruction of the properties of the individual phases from the measurable diffusion averages, which is an important requirement regarding the further development of diagnostic techniques for in vivo optical measurement of bone quality.

\section{Acknowledgments}

EMB's work was funded by STW, project no 7505. The authors want to thank Dr ir. A J Veen and Dr ir. Th H Smit for valuable discussions and support with sample acquisition.

\section{References}

Akaike H 1974 A new look at the statistical model identification IEEE Trans. Autom. Control 19 716-23 Arridge S R, Cope M and Delpy D T 1992 The theoretical basis for the determination of optical pathlengths in tissue: temporal and frequency analysis Phys. Med. Biol. 37 1531-60

Ascenzi A and Fabry C 1959 Technique for dissection and measurement of refractive index of osteones $J$. Cell Biol. 6 139-42

Bal H and Verstoep K 2008 Large-scale parallel computing on grids Electron. Notes Theor. Comput. Sci. 220 3-17

Bensoussan A, Lions J L and Papanicolaou G C 1979 Boundary layers and homogenization of transport processes Publ. Res. Inst. Math. Sci. 15 53-157

Dumas L and Golse F 2000 Homogenization of transport equations SIAM J. Appl. Math. 60 1447-70 
Firbank M, Hiraoka M, Essenpreis M and Delpy D T 1993 Measurement of the optical properties of the skull in the wavelength range 650-950 nm Phys. Med. Biol 38 503-10

Firbank M, Okada E and Delpy D T 1997 Investigation of the effect of discrete absorbers upon the measurement of blood volume with near-infrared spectroscopy Phys. Med. Biol. 42 465-77

Garland M and Heckbert P 1997 Surface simplification using quadric error metrics Proc. SIGGRAPH

Hebden J C and Austin T 2007 Optical tomography of the neonatal brain Eur. Radiol. 17 2926-33

Hebden J C, García Guerrero J J, Chernomordik V and Gandjbakhche A H 2004 Experimental evaluation of an anisotropic scattering model of a slab geometry Opt. Lett. 29 2518-20

Hildebrand T and Rüegsegger P 1997 A new method for the model-independent assessment of thickness in threedimensional images J. Microsc. 185 67-75

King A B, Saag K G, Burge R T, Pisu M and Goel N 2005 Fracture reduction affects Medicare economics (FRAME): impact of increased osteoporosis diagnosis and treatment Osteoporos. Int. 16 1545-57

Lev A, Rubanov E, Sfez B, Shany S and Foldes A J 2005 Ultrasound-modulated light tomography assessment of osteoporosis Opt. Lett. $301692-4$

Liu H, Chance B, Hielscher A H, Jacques S L and Tittel F K 1995 Influence of blood vessels on the measurement of hemoglobin oxygenation as determined by time-resolved reflectance spectroscopy Med. Phys. 22 1209-17

Margallo-Balbás E and French P J 2007 Shape based Monte Carlo code for light transport in complex heterogeneous tissues Opt. Express 15 14086-98

Odgaard A and Gundersen H J 1993 Quantification of connectivity in cancellous bone, with special emphasis on 3-D reconstruction Bone 14 173-82

Padilla F, Bossy E and Laugier P 2006 Simulation of ultrasound propagation through three-dimensional trabecular bone structures: comparison with experimental data Japan. J. Appl. Phys. 456496

Padilla F, Jenson F, Bousson V, Peyrin F and Laugier P 2008 Relationships of trabecular bone structure with quantitative ultrasound parameters: in vitro study on human proximal femur using transmission and backscatter measurements Bone 42 1193-202

Pavliotis G A and Stuart A M 2008 Multiscale Methods: Averaging and Homogenization (New York: Springer)

Pifferi A, Torricelli A, Taroni P, Bassi A, Chikoidze E, Giambattistelli E and Cubeddu R 2004 Optical biopsy of bone tissue: a step toward the diagnosis of bone pathologies J. Biomed. Opt. 9 474-9

Sassaroli A, Martelli F, Imai D and Yamada Y 1999 Study on the propagation of ultra-short pulse light in cylindrical optical phantoms Phys. Med. Biol. 44 2747-63

Takeuchi A, Araki R, Proskurin S G, Takahashi Y, Yamada Y, Ishii J, Katayama S and Tiabashi A 1997 A new method of bone tissue measurement based upon light scattering J. Bone Miner. Res. 12 261-6

Talsma A, Chance B and Graaff R 2001 Corrections for inhomogeneities in biological tissue caused by blood vessels J. Opt. Soc. Am. A 18 932-9

Tanaka S M, Kakio M and Yamakoshi K-i 2008 Non-destructive optical monitoring for calcification of tissueengineered bone in vitro J. Biomech. Sci. Eng. 3 332-42

Ugryumova N, Matcher S J and Attenburrow D P 2004 Measurement of bone mineral density via light scattering Phys. Med. Biol. 49 469-83

van Veen R L P, Verkruysse W and Sterenborg H J C M 2002 Diffuse-reflectance spectroscopy from 500 to $1060 \mathrm{~nm}$ by correction for inhomogeneously distributed absorbers Opt. Lett. 27 246-8

Vo-Dinh T (ed) 2003 Biomedical Photonics Handbook (Boca Raton, FL: CRC Press)

Yang S-M, Shin S-Y and Kye S-B 2008 Relationship between implant stability measured by resonance frequency analysis (RFA) and bone loss during early healing period Oral Surg. Oral Med. Oral Pathol. Oral Radiol. Endod. 105 e12-19

Zhao H, Gao F, Tanikawa Y, Homma K and Yamada Y 2005 Time-resolved diffuse optical tomographic imaging for the provision of both anatomical and functional information about biological tissue Appl. Opt. 44 1905-16 